\title{
Nonequilibrium chiral perturbation theory and pion decay functions
}

\author{
A. Gómez Nicola ${ }^{\mathrm{a}, 1}$, V. Galán-González b,2 \\ ${ }^{a}$ Departamento de Física Teórica, Universidad Complutense, 28040 Madrid, Spain \\ b Theoretical Physics, Blackett Laboratory, Imperial College, Prince Consort Road, London SW7 2BZ, UK \\ Received 1 December 1998 \\ Editor: R. Gatto
}

\begin{abstract}
We extend chiral perturbation theory to study a meson gas out of thermal equilibrium. Assuming that the system is initially in equilibrium at $T_{i}<T_{c}$ and working within the Schwinger-Keldysh contour technique, we define consistently the time-dependent temporal and spatial pion decay functions, the counterparts of the pion decay constants, and calculate them to next to leading order. The link with curved space-time QFT allows to establish nonequilibrium renormalisation. The short-time behaviour and the applicability of our model to a heavy-ion collision plasma are also discussed in this work. (C) 1999 Elsevier Science B.V. All rights reserved.
\end{abstract}

PACS: 12.39.Fe; 11.10.Wx; 05.70.Ln; 12.38.Mh

Keywords: Nonequilibrium thermal field theory; Chiral perturbation theory; Pion decay constants; Heavy-ion collisions; Quantum field theory in curved space-time

\section{Introduction}

The chiral phase transition plays a fundamental role in the description of the plasma formed after a relativistic heavy-ion collision (RHIC), where it is imperative to use meson effective models to describe QCD. Two of the most successful approaches are the $O(4)$ linear sigma model (LSM), valid only for $N_{f}=2$ light flavours, and Chiral Perturbation Theory (ChPT), based on derivative expansions compatible with the QCD symmetries, and whose lowest order action is the nonlinear sigma model (NLSM) [1]. In ChPT, the perturbative parameter is $p / \Lambda_{\chi}$, with $p$ a meson energy (like masses, external momenta or temperature) and $\Lambda_{\chi} \simeq 1 \mathrm{GeV}$. Every meson loop is $\mathscr{O}\left(p^{2} / \Lambda_{\chi}^{2}\right)$ and all the infinities coming from them can be absorbed in the coefficients of higher order lagrangians $[1,2]$.

In thermal equilibrium at finite temperature $T$, the chiral symmetry is believed to be restored at $T_{c} \simeq$ 150-200 MeV [3]. In fact, near $T_{c}$, the mean-field LSM is well known to undergo a second-order phase

\footnotetext{
${ }^{1}$ E-mail: gomez@eucmax.sim.ucm.es

${ }^{2}$ E-mail: v.galan-gonz@ic.ac.uk
} 
transition. The NLSM is equally valid for reproducing the phase transition, provided one works in the large- $N$ limit [4]. Strictly within ChPT, the low-temperature meson gas has been studied [5,6] on expansion in $T^{2} / \Lambda_{\chi}^{2}$, predicting the correct behaviour of the observables as $T$ approaches $T_{c}$.

The equilibrium assumption is not realistic if one is interested in the dynamics of the expanding plasma formed after a RHIC, where several nonequilibrium effects could be important. One of them is the formation of disoriented chiral condensates (DCC), regions in which the chiral field is correlated and has nonzero components in the pion direction [7]. As the plasma expands, long-wavelength pion modes — propagating as if they had an effective negative mass squared- can develop instabilities growing fast as the field relaxes to the ground state, an observable consequence being coherent pion emission [8]. This issue has been extensively studied in the literature, mostly within the LSM assuming initial thermal equilibrium at $T_{i}>T_{c}$, either encoding the cooling mechanism in the time dependence of the lagrangian parameters [8-11] or describing the plasma expansion in proper time and rapidity [12]. This phenomenon has also been studied using Gross-Neveu models [13]. Another important nonequilibrium observable is the photon and dilepton production [14], to which the anomalous meson sector could significantly contribute [10].

In this work we will construct an effective ChPT-based model to describe a meson gas out of thermal equilibrium, as an alternative to the LSM approach. Our only degrees of freedom will be then the Nambu-Goldstone bosons (NGB) and we will consider the most general low-energy lagrangian compatible with the QCD symmetries. We will restrict here to $N_{f}=2$ (where the NGB are just the pions) and to the chiral limit (massless quarks), which is the simplest approximation allowing to build the model in terms of exact chiral symmetry. One of the novelties of our approach is to exploit the analogy between ChPT and the physical regime where the system is not far from equilibrium and then a derivative expansion is consistent.

\section{The NLSM and ChPT out of equilibrium}

We will take the system in thermal equilibrium for $t \leq 0$ at a temperature $T_{i}<T_{c}$ and for $t>0$ we let the lagrangian parameters be time-dependent. We are also assuming that the system is homogeneous and isotropic. The generating functional of the theory can then be formulated in the path integral formalism, by letting the time integrals run over the Schwinger-Keldysh contour $C$ displayed in Fig. 1 [15-18]. We will eventually let $t_{i} \rightarrow-\infty$ and $t_{f} \rightarrow+\infty$, although we will show that our results are independent of $t_{i}$ and $t_{f}$. We remark that, even in that limit, the imaginary-time leg of $C$ has to be kept, since it encodes the KMS equilibrium boundary conditions [17-19]. With these assumptions, our low-energy model will be the following nonequilibrium NLSM

$$
S[U]=\int_{C} d^{4} x \frac{f^{2}(t)}{4} \operatorname{tr} \partial_{\mu} U^{\dagger}(\boldsymbol{x}, t) \partial^{\mu} U(\boldsymbol{x}, t)
$$

where $\int_{C} d^{4} x \equiv \int_{C} d t \int d^{3} \boldsymbol{x}, U(\boldsymbol{x}, t) \in S U(2)$ is the NGB field, satisfying $U\left(\boldsymbol{x}, t_{i}+i \beta_{i}\right)=U\left(\boldsymbol{x}, t_{i}\right)$ with $\beta_{i}=T_{i}^{-1}$, and $f(t)$ is a real function which in equilibrium and to the lowest order (see Section 4) would be $f=f_{\pi} \simeq 93$ $\mathrm{MeV}$ (the pion decay constant) i.e, $f(t \leq 0)=f$. Note that $f(t)$ cannot be analytic at $t=0$ and, in particular, it

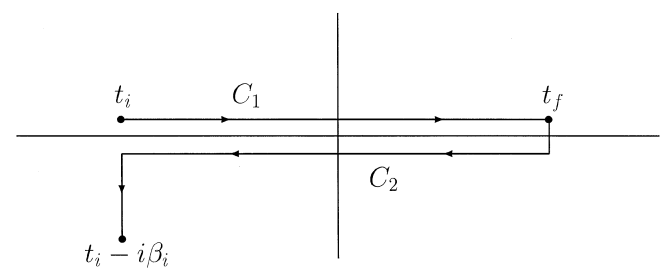

Fig. 1. The contour $C$ in complex time $t$. The lines $C_{1}$ and $C_{2}$ run between $t_{i}+i \epsilon$ and $t_{f}+i \epsilon$ and $t_{f}-i \epsilon$ and $t_{i}-i \epsilon$ respectively, with $\epsilon \rightarrow 0^{+}$. 
could be discontinuous, like the meson mass in quenched LSM approaches [8-11]. This is a consequence of the nature of our approach, since the system is driven off equilibrium instantaneously. An alternative, which we will not attempt here, is to choose $f(t)$ analytic $\forall t$, having equilibrium only at $t=t_{i}$ [20]. Thus, the temporal evolution of our results will start at $t=0^{+}$, an infinitesimally small response time. As we will see below, our approach is consistent because the discontinuities at $t=0$ appear to NLO.

We will parametrise the field $U$ as

$$
U(\boldsymbol{x}, t)=\frac{1}{f(t)}\left\{\left[f^{2}(t)-\pi^{2}(\boldsymbol{x}, t)\right]^{1 / 2} I+i \tau_{a} \pi^{a}(\boldsymbol{x}, t)\right\} ; \quad a=1,2,3,
$$

where $\pi^{2}=\pi^{a} \pi_{a}, \pi^{a}$ the pion fields satisfying $\pi^{a}\left(t_{i}+i \beta_{i}\right)=\pi^{a}\left(t_{i}\right)$ and $I$ and $\tau_{a}$ are the identity and Pauli matrices. Note that with the choice (2) we recover the canonical kinetic term in the action after expanding $U$ in powers of $\pi$. Other choices amount to a time-dependent normalisation of the pion fields and should not have any effect on the physics (see Section 4). For instance, if we redefine $\tilde{\pi}^{a}=\pi^{a} f(0) / f(t)$, the action for the $\tilde{\pi}^{a}(\boldsymbol{x}, t)$ fields is just the equilibrium NLSM multiplied by the time-dependent scale factor $f^{2}(t) / f^{2}(0)$ (see below).

Our action (1) is manifestly chiral invariant $\left(U(x) \rightarrow L U(x) R^{\dagger}\right)$. Notice that we work in the chiral limit and hence there are no explicit symmetry-breaking pion mass terms in the action. The conserved axial and vector currents for the chiral symmetry can be derived by applying the standard procedure [1,2], so that the axial current reads

$$
A_{\mu}^{a}(\boldsymbol{x}, t)=i \frac{f^{2}(t)}{4} \operatorname{tr}\left[\tau^{a}\left(U^{\dagger} \partial_{\mu} U-U \partial_{\mu} U^{\dagger}\right)\right]
$$

Let us now discuss how to establish a consistent nonequilibrium ChPT. The new ingredient we need is the temporal variation of $f(t)$. We will then consider

$$
\frac{\dot{f}(t)}{f^{2}(t)} \simeq \mathscr{O}\left(\frac{p}{\Lambda_{\chi}}\right), \quad \frac{\ddot{f}(t)}{f^{3}(t)}, \frac{[\dot{f}(t)]^{2}}{f^{4}(t)} \simeq \mathscr{O}\left(\frac{p^{2}}{\Lambda_{\chi}^{2}}\right),
$$

and so on, the rest of the chiral power counting being the same as in equilibrium. Therefore, in our approach we treat the deviations of the system from equilibrium perturbatively, following the ChPT guidelines. Thus, we will expand our action (1) to the relevant order in pion fields and take into account all the contributing Feynman diagrams. The loop divergences should be such that they can be absorbed in the coefficients of higher order lagrangians, which in general will require the introduction of new time-dependent counterterms (see below). Notice also that according to (4), we can always describe the short-time nonequilibrium regime, just by expanding $f(t)$ around $t=0^{+}$. In fact, for times $t \leq f_{\pi}^{-1}$, that is equivalent to a chiral expansion, since then $\dot{f}\left(0^{+}\right) t / f\left(0^{+}\right)=\mathscr{O}\left(p / \Lambda_{\chi}\right)$ and so on. Nonetheless, we stress that the conditions (4) do not imply working at short-times, but just to remain close enough to equilibrium.

To leading order in $\pi$ fields, the action (1), after using (2), reads

$$
S_{0}[\pi]=-\frac{1}{2} \int_{C} d^{4} x \pi^{a}(\boldsymbol{x}, t)\left[\square+m^{2}(t)\right] \pi^{a}(\boldsymbol{x}, t) \quad \text { with } m^{2}(t)=-\frac{\ddot{f}(t)}{f(t)},
$$

where we have partial integrated in $C$. Thus, the leading order nonequilibrium effect of our model can be written as a time-dependent pion mass term, which, as commented before, is a common feature of nonequilibrium models [9-12]. Notice that $m^{2}(t)$ can be negative, so that our model accommodates unstable pion modes, 
whose importance we have discussed before. Note also that this mass term does not break the chiral symmetry, i.e, the axial current is classically conserved. Indeed, to leading order we have, from (3),

$$
\left[A_{\mu}^{a}(\boldsymbol{x}, t)\right]^{\mathrm{LO}}=-f(t) \partial_{\mu} \pi^{a}(\boldsymbol{x}, t)+\delta_{\mu 0} \dot{f}(t) \pi^{a}(\boldsymbol{x}, t),
$$

which satisfies $\partial^{\mu} A_{\mu}=0$ using $\left[\square+m^{2}(t)\right] \pi^{a}=0$, the equations of motion to the same order. Had we included the pion mass term $m_{\pi}$ - explicitly breaking the symmetry - the instabilities threshold, to leading order, would have been $m^{2}(t)<-m_{\pi}^{2}$ instead.

It is very interesting to rephrase our model as a NLSM in a curved space-time background $g_{\mu \nu}$, which reads [21,22],

$$
S_{g}[U]=\frac{f^{2}(0)}{4} \int_{C} d^{4} x(\sqrt{-g}) g^{\mu \nu} \operatorname{tr} \partial_{\mu} U^{\dagger}(x) \partial_{\nu} U(x)+\xi S_{R}[U, R]
$$

plus $U$ independent terms, where $g=\operatorname{det} g$ and the last term accounts for possible couplings between the pion fields and the scalar curvature $R(x)$ (like $R(x) \phi^{2}$ for a free scalar field $\phi$ [21]). Now, notice that our nonequilibrium model (1) is obtained by writing $U(x)$ in the $\tilde{\pi}$ parametrisation discussed before (i.e, with $f(t)$ replaced by $f(0)$ in (2)), choosing $\xi=0$ (minimal coupling) and a spatially flat Robertson-Walker (RW) space-time in conformal time, whose line element is $d s^{2}=a^{2}(\eta)\left[d \eta^{2}-d x^{2}\right]$, with the scale factor $a(\eta)=$ $f(\eta) / f(0)$. Our effective theory is then not only suitable for a RHIC environment, but also in a cosmological framework. Notice also that if we take $\xi \neq 0$, the lowest order $S_{R}$ term we can construct has the form of an effective mass term breaking explicitly the chiral symmetry. In fact, it is not difficult to see that we could cancel the $m^{2}(t)$ term in (5) by choosing $\xi=1 / 6$, which is the value rendering the theory scale invariant [21]. This is just a consequence of the lagrangian chiral and conformal symmetries being incompatible in a curved background [22] or, equivalently, at nonequilibrium. In other words, for $\xi=0$-which is our choice, since we want to preserve chiral symmetry, as in Ref. [22] — we may interpret the $m^{2}(t)$ term, in the chiral limit, as the minimal coupling with the background yielding chiral invariance.

The above equivalence turns out to be very useful to renormalise our model, consistently with ChPT. In fact, all the one-loop divergences arising from (7) can be absorbed in the coefficients of the $\mathscr{O}\left(p^{4}\right)$ action $S_{4}$, which consists of the Minkowski terms with indices raised and lowered with $g_{\mu \nu}$ plus new chiral-invariant couplings of pion fields with the curvature [22]. In the chiral limit, those new terms read

$$
\begin{aligned}
S_{4}^{R}[\pi] & =\int_{C} d^{4} x(\sqrt{-g})\left[L_{11} R(x) g^{\mu \nu}+L_{12} R^{\mu \nu}\right] \operatorname{tr} \partial_{\mu} U^{\dagger}(x) \partial_{\nu} U(x) \\
& =-\frac{1}{2} \int_{C} d^{4} x \pi^{a}\left[f_{1}(t) \partial_{t}^{2}-f_{2}(t) \nabla^{2}+m_{1}^{2}(t)\right] \pi^{a}+\mathscr{O}\left(\pi^{4}\right)
\end{aligned}
$$

where $R_{\mu \nu}$ is the Ricci tensor, $L_{11}$ and $L_{12}$ are two new low-energy constants [22] and we have given the two-pion contributions in the parametrisation (2), after partial integration, with our RW metric, where

$$
\begin{aligned}
& f_{1}(t)=12\left[\left(2 L_{11}+L_{12}\right) \frac{\ddot{f}(t)}{f^{3}(t)}-L_{12} \frac{[\dot{f}(t)]^{2}}{f^{4}(t)}\right], \\
& f_{2}(t)=4\left[\left(6 L_{11}+L_{12}\right) \frac{\ddot{f}(t)}{f^{3}(t)}+L_{12} \frac{[\dot{f}(t)]^{2}}{f^{4}(t)}\right], \\
& m_{1}^{2}(t)=-\left[\frac{f_{1}(t) \ddot{f}(t)+\dot{f_{1}}(t) \dot{f}(t)}{f(t)}+\frac{1}{2} \ddot{f}_{1}(t)\right] .
\end{aligned}
$$

for $t>0$ and $f_{i}(t \leq 0)=0$. The above terms are the only ones in $S_{4}$ containing two pions and they will renormalise purely nonequilibrium infinities — which are time-dependent and vanish for $t \leq 0-$. It is important 
to bear in mind that to cancel the one-loop new divergences only $L_{11}$ needs to be renormalised, whereas $L_{12}=L_{12}^{r}$ [22]. We will come back to this point below.

Next, we will concentrate on the Green functions time-ordered along $C[17,18]$. Unless otherwise stated, we will be using the parametrisation (2) in the remaining of this work. The two-point function defines the pion propagator $G^{a b}(x, y)=-i\left\langle T_{C} \pi^{a}(x) \pi^{a}(y)\right\rangle$, which to leading order $G_{0}^{a b}(x, y)=\delta^{a b} G_{0}(x, y)$, by isospin invariance, and

$$
\left\{\square_{x}+m^{2}\left(x^{0}\right)\right\} G_{0}(x, y)=-\delta_{C}\left(x^{0}-y^{0}\right) \delta^{(3)}(\boldsymbol{x}-\boldsymbol{y})
$$

with KMS equilibrium conditions $G_{0}^{>}\left(\boldsymbol{x}, t_{i}-i \beta_{i} ; y\right)=G_{0}^{<}\left(\boldsymbol{x}, t_{i} ; y\right)$, the advanced and retarded propagators being defined as customarily along $C$. Notice that $G\left(x, x^{\prime}\right)=G\left(t, t^{\prime}, \boldsymbol{x}-\boldsymbol{x}^{\prime}\right)$ due to the nonequilibrium lack of time translation invariance. Therefore, we will define, as customarily, the "fast" temporal variable $t-t^{\prime}$ and the "slow" one $\tau \equiv\left(t+t^{\prime}\right) / 2$, so that $F\left(q_{0}, \omega_{q}, \tau\right)$ and $F\left(\omega_{q}, t, t^{\prime}\right)$, with $\omega_{q}^{2}=|\boldsymbol{q}|^{2}$, will denote, respectively, the fast and mixed (in which only the spatial coordinates are transformed) Fourier transforms of $F\left(x, x^{\prime}\right)$. Note that $F\left(q_{0}, \omega_{q}, \tau\right)$ depends separately on $q_{0}$ and $\omega_{q}$ because of the thermal loss of Lorentz covariance and has the extra nonequilibrium $\tau$-dependence. Then, in the mixed representation, (10) becomes

$$
\left[\frac{d^{2}}{d t^{2}}+\omega_{q}^{2}+m^{2}(t)\right] G_{0}\left(\omega_{q}, t, t^{\prime}\right)=-\delta_{C}\left(t-t^{\prime}\right)
$$

The general solution of (11) is only known explicitly for some particular choices of $m^{2}(t)[21,17,18]$. Formally, we can write it as a Schwinger-Dyson equation as

$$
G_{0}\left(\omega_{q}, t, t^{\prime}\right)=G_{0}^{e q}\left(\omega_{q}, t-t^{\prime}\right)+\int_{C} d z m^{2}(z) G_{0}^{e q}\left(\omega_{q}, t-z\right) G_{0}\left(\omega_{q}, z, t^{\prime}\right)
$$

with $G_{0}^{e q}\left(\omega_{q}, t-t^{\prime}\right)$ the equilibrium solution of (11), i.e, with $m^{2}(t)=0$.

Another object of interest for our purposes is the Lehman spectral function $\rho(x, y)=G^{>}(x, y)-G^{<}(x, y)$ [16], which in equilibrium to leading order is $\rho_{0}^{e q}(q)=-2 \pi i \operatorname{sgn}\left(q_{0}\right) \delta\left(q^{2}\right)$ [19]. Note that, by construction, $G^{>}(x, y)=G^{<}(y, x)$, so that $\rho(x, y)=-\rho(y, x)$ and $\rho\left(q_{0}, \omega_{q}, \tau\right)=-\rho\left(-q_{0}, \omega_{q}, \tau\right)$. The normalisation of $\rho_{0}$ is

$$
\frac{1}{2 \pi i} \int_{-\infty}^{+\infty} q_{0} \rho_{0}\left(q_{0}, \omega_{q}, \tau\right)=\left.\frac{d \rho_{0}\left(\omega_{q}, t, t^{\prime}\right)}{d t}\right|_{t=t^{\prime}}=-1,
$$

which can be readily checked by using (12) and $\rho_{0}\left(\omega_{q}, t, t\right)=0$.

\section{Next to leading order propagator}

We will now obtain the NLO correction to the propagator. For that purpose, we need the action in (1) up to four-pion terms:

$$
S[\pi]=S_{0}[\pi]+\frac{1}{2} \int_{C} d^{4} x\left\{\frac{1}{f^{2}(t)}\left[\partial_{\mu} \pi^{a} \partial^{\mu} \pi^{b} \pi_{a} \pi_{b}+\frac{1}{2}\left(\pi^{2}\right)^{2}\left(\frac{\ddot{f}(t)}{f(t)}-\frac{\dot{f}^{2}(t)}{f^{2}(t)}\right)\right]+\mathscr{O}\left(\pi^{6}\right)\right\}
$$

plus the two-pion terms in (8). The two diagrams contributing are, respectively, (a) and (b) in Fig. 2.

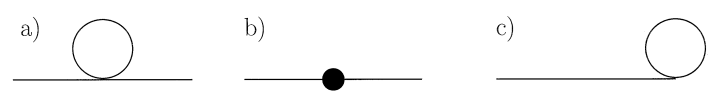

Fig. 2. Diagrams contributing to the NLO pion propagator (a,b) and axial-axial correlator (c). The black dot in b) represents the interaction coming from $S_{4}^{R}$ in (8). 
Let us concentrate on $G_{11}$ (i.e, $t, t^{\prime} \in C_{1}$ in Fig. 1) and $t$ and $t^{\prime}$ positive, which is the relevant case for our purposes, as commented above. We will use dimensional regularisation (DR), so that evaluating the above diagrams, using $(10)$ with $\delta^{(d)}(0)=0$, and after some algebra, we obtain in the mixed representation to NLO

$$
\begin{aligned}
G_{11}^{>}\left(t, t^{\prime}\right)= & G_{0,11}^{>}\left(t, t^{\prime}\right)\left(1-\frac{1}{2}\left[f_{1}(t)+f_{1}\left(t^{\prime}\right)\right]\right)+\frac{i}{2 \omega_{q}} \operatorname{coth}\left[\frac{\beta_{i} \omega_{q}}{2}\right] \frac{T_{i}^{2}}{12 f^{2}} \cos \left[\omega_{q}\left(t+t^{\prime}\right)\right] \\
& +i\left\{\int_{0}^{t} d \tilde{t}\left[\Delta_{1}\left(\tilde{t}, \omega_{q}\right) G_{0}^{>}(\tilde{t}, t) G_{0}^{>}\left(\tilde{t}, t^{\prime}\right)+\Delta_{2}(\tilde{t}) \dot{G}_{0}^{>}(\tilde{t}, t) \dot{G}_{0}^{>}\left(\tilde{t}, t^{\prime}\right)\right]\right. \\
& -\int_{0}^{t^{\prime}} d \tilde{t}\left[\Delta_{1}\left(\tilde{t}, \omega_{q}\right) G_{0}^{<}(\tilde{t}, t) G_{0}^{<}\left(\tilde{t}, t^{\prime}\right)+\Delta_{2}(\tilde{t}) \dot{G}_{0}^{<}(\tilde{t}, t) \dot{G}_{0}^{<}\left(\tilde{t}, t^{\prime}\right)\right] \\
& \left.-\int_{t^{\prime}}^{t} d \tilde{t}\left[\Delta_{1}\left(\tilde{t}, \omega_{q}\right) G_{0}^{<}(\tilde{t}, t) G_{0}^{>}\left(\tilde{t}, t^{\prime}\right)+\Delta_{2}(\tilde{t}) \dot{G}_{0}^{<}(\tilde{t}, t) \dot{G}_{0}^{>}\left(\tilde{t}, t^{\prime}\right)\right]\right\}
\end{aligned}
$$

and $G_{11}^{<}\left(t, t^{\prime}\right)=G_{11}^{>}\left(t^{\prime}, t\right)$, where we have suppressed for simplicity the $\omega_{q}$ dependence of the propagators, the dot denotes $d / d \tilde{t}$,

$$
\begin{aligned}
& \Delta_{1}\left(\tilde{t}, \omega_{q}\right)=\frac{1}{f^{2}(\tilde{t})}\left[\left(6 \frac{\ddot{f}(\tilde{t})}{f(\tilde{t})}-5\left(\frac{\dot{f}(\tilde{t})}{f(\tilde{t})}\right)^{2}-\omega_{q}^{2}\right) G_{0}(\tilde{t})-2 \ddot{G}_{0}(\tilde{t})+4 \frac{\dot{f}(\tilde{t})}{f(\tilde{t})} \dot{G}_{0}(\tilde{t})\right] \\
& +i \omega_{q}^{2}\left[f_{2}(\tilde{t})-f_{1}(\tilde{t})\right]-i\left[\frac{\dot{f_{1}}(\tilde{t}) \dot{f}(\tilde{t})}{f(\tilde{t})}+\frac{1}{2} \ddot{f}_{1}(\tilde{t})\right], \\
& \Delta_{2}(\tilde{t})=\frac{G_{0}(\tilde{t})}{f^{2}(\tilde{t})}
\end{aligned}
$$

and $G_{0}\left(z^{0}\right) \equiv G_{0}(z, z)$ is the equal-time correlation function. We observe that (15) is $t_{i}$ and $t_{f}$ independent, which is a good consistency check. Notice also that by replacing the equilibrium propagators in (15), we recover

$$
G_{11}^{e q>}\left(t-t^{\prime}\right)=G_{0,11}^{e q>}\left(t-t^{\prime}\right)\left(1-\frac{T^{2}}{12 f^{2}}\right)
$$

which agrees with [4] (note that we have derived it for the contour $C$, including both imaginary-time and real-time thermal field theory) and is finite in the chiral limit, where there is no tadpole renormalisation in DR [2]. However, out of equilibrium, the NLO propagator is in general divergent, even in the chiral limit, and the infinities have to be absorbed in the two-pion counterterms in (8).

\section{The nonequilibrium pion decay functions}

In a thermal bath, the concepts of LSZ and asymptotic states are subtle, and so is then the extension of low-energy theorems like PCAC. Thus, pion decay constants are more conveniently defined through the thermal axial-axial correlator $A_{\mu \nu}^{a b}(x, y)=\left\langle T_{C} A_{\mu}^{a}(x) A_{\mu}^{b}(y)\right\rangle$. At $T \neq 0$ the loss of Lorentz covariance in the tensorial structure of $A_{\mu \nu}$ implies that one can define two independent and complex $f_{\pi}^{s}$ (spatial) and $f_{\pi}^{t}$ (temporal), their 
real and imaginary parts being related respectively with the pion velocity and damping rate in the thermal bath [23]. Nevertheless, to one-loop in the chiral limit one has $[5,4,23]$

$$
\left[f_{\pi}^{s}(T)\right]^{2}=\left[f_{\pi}^{t}(T)\right]^{2}=f^{2}\left(1-\frac{T^{2}}{T_{c}^{2}}\right),
$$

with $T_{c}=\sqrt{6} f_{\pi} \simeq 228 \mathrm{MeV}$. Despite it being just the lowest order in the low temperature expansion, (19) predicts the right behaviour and a reasonable estimate for the critical temperature, although, strictly speaking, $f_{\pi}(T)$ is not the order parameter [4]. To higher orders, $f_{\pi}^{s} \neq f_{\pi}^{t}$ and $\operatorname{Im} f_{\pi}^{s, t} \neq 0$ [23].

Let us then analyze $A_{\mu \nu}^{a b}$ in our nonequilibrium model. The relevant quantity, as far as $f_{\pi}$ is concerned, is the spectral function $\rho_{\mu \nu}=A_{\mu \nu}^{>}-A_{\mu \nu}^{<}$, with $A_{\mu \nu}^{a b}=\delta^{a b} A_{\mu \nu}$. We readily realise that $\rho_{\mu \nu}\left(q_{0}, \boldsymbol{q}, \tau\right)=-\rho_{\nu \mu}\left(-q_{0},-\right.$ $\boldsymbol{q}, \tau)$. Then, from rotational symmetry,

$$
\rho_{i j}\left(q_{0}, \boldsymbol{q}, \tau\right)=q_{i} q_{j} \rho_{L}\left(q_{0}, \omega_{q}, \tau\right)+\delta_{i j} \rho_{d}\left(q_{0}, \omega_{q}, \tau\right)
$$

with $\rho_{L, d}\left(q_{0}\right)=-\rho_{L, d}\left(-q_{0}\right)^{3}$ and $\rho_{j 0}\left(q_{0}, \boldsymbol{q}, \tau\right)=q_{j} \rho_{S}\left(q_{0}, \omega_{q}, \tau\right)$. Therefore, $\rho_{\mu \nu}$ is characterized, in principle by the four functions $\rho_{L}, \rho_{d}, \rho_{S}$ and $\rho_{00}$. However, they are related through the $A_{\mu}$ conservation Ward Identity (WI) $\partial_{\mu}^{x} \rho^{\mu \nu}(x, y)=\partial_{\nu}^{y} \rho^{\mu \nu}(x, y)=0$, which also holds in our model. Thus, we get

$$
\begin{aligned}
& q^{0} \rho_{00}\left(q_{0}, \omega_{q}, \tau\right)-\omega_{q}^{2} \rho_{S}\left(q_{0}, \omega_{q}, \tau\right)-\frac{i}{2} \dot{\rho}_{00}\left(q_{0}, \omega_{q}, \tau\right)=0, \\
& q^{0} \rho_{S}\left(q_{0}, \omega_{q}, \tau\right)-\omega_{q}^{2} \rho_{L}\left(q_{0}, \omega_{q}, \tau\right)+\frac{i}{2} \dot{\rho}_{S}\left(q_{0}, \omega_{q}, \tau\right)+\rho_{d}\left(q_{0}, \omega_{q}, \tau\right)=0,
\end{aligned}
$$

where the dot denotes $\partial / \partial \tau$. Thus, only two components of $\rho_{\mu \nu}$ are independent, as in equilibrium [4], where there are no time derivatives in the above equation.

At $T=0$ one has $\rho_{L}=2 \pi f_{\pi}^{2} \operatorname{sgn}\left(q^{0}\right) \delta\left(q^{2}\right)$, since there exist NGB states. That is not the case at $T \neq 0$, where the pion dispersion relation is not in general a $\delta$-function [4]. In fact, to define properly $f_{\pi}(T)$ requires taking the $\omega_{q} \rightarrow 0^{+}$limit, in which a zero-energy excitation still exists [4], although to NLO there is no need to take that limit. Extending these ideas to nonequilibrium, we will define the time-dependent pion decay functions (PDF) as

$$
\begin{aligned}
& {\left[f_{\pi}^{s}(t)\right]^{2}=\frac{1}{2 \pi} \lim _{\omega_{q} \rightarrow 0^{+}} \int_{-\infty}^{\infty} d q_{0} q_{0} \rho_{L}\left(q_{0}, \omega_{q}, t\right)=\left.\lim _{\omega_{q} \rightarrow 0^{+}} i \frac{d}{d t} \rho_{L}\left(\omega_{q}, t, t^{\prime}\right)\right|_{t=t^{\prime}}} \\
& f_{\pi}^{s}(t) f_{\pi}^{t}(t)=\frac{1}{2 \pi} \lim _{\omega_{q} \rightarrow 0^{+}} \int_{-\infty}^{\infty} d q_{0} \rho_{S}\left(q_{0}, \omega_{q}, t\right)=\lim _{\omega_{q} \rightarrow 0^{+}} \rho_{S}\left(\omega_{q}, t, t\right) \\
& f_{\pi}^{s}(t) g_{\pi}(t)=-\frac{i}{2 \pi} \lim _{\omega_{q} \rightarrow 0^{+}} \int_{-\infty}^{\infty} d q_{0} q_{0} \rho_{S}\left(q_{0}, \omega_{q}, t\right)=\left.\frac{1}{2} \lim _{\omega_{q} \rightarrow 0^{+}} \frac{d}{d t} \rho_{S}\left(\omega_{q}, t, t^{\prime}\right)\right|_{t=t^{\prime}}
\end{aligned}
$$

The functions $f_{\pi}^{s}(t)$ and $f_{\pi}^{t}(t)$ are the nonequilibrium counterparts of the spatial and temporal pion decay constants respectively, whereas $g_{\pi}(t)$ vanishes in equilibrium. However, the above PDF are related through the WI. Integrating in $q_{0}$ in (21), we get

$$
f_{\pi}^{s}(t) g_{\pi}(t)=\frac{1}{2} \frac{d}{d t}\left[f_{\pi}^{t}(t) f_{\pi}^{s}(t)\right]
$$

so that only two PDF are independent, as in equilibrium [23]. Let us now check the consistency of our definitions to leading order. From $(6), \rho_{L}^{\mathrm{LO}}\left(\omega_{q}, t, t^{\prime}\right)=i f(t) f\left(t^{\prime}\right) \rho_{0}\left(\omega_{q}, t, t^{\prime}\right)$ and $\rho_{d}=0$, so that, using (13) yields

\footnotetext{
${ }^{3}$ Our $\rho_{L}$ and $\rho_{d}$ correspond in in the notation of [4], to $\operatorname{sgn}\left(q^{0}\right) \rho_{A}^{L} q_{0}^{2} / \omega_{q}^{2} q^{2}$ and $\operatorname{sgn}\left(q^{0}\right) \rho_{A}^{T}$ respectively.
} 
$f_{\pi}^{s}(t)_{L O}^{2}=f^{2}(t)$, i.e, the PDF coincides with $f(t)$ to leading order, as it should be. Similarly, we find $f_{\pi}^{t}(t)_{L O}=f_{\pi}^{s}(t)_{L O}=f(t)$ and $g_{\pi}(t)_{L O}=f(t)$, so that our definitions are consistent to leading order.

To NLO, we need the axial current up to $\mathscr{O}\left(\pi^{3}\right)$. From (3),

$$
A_{\mu}^{a}(\boldsymbol{x}, t)=\left[A_{\mu}^{a}(\boldsymbol{x}, t)\right]^{\mathrm{LO}}-\frac{1}{2 f(t)}\left(\pi^{a} \partial_{\mu} \pi^{2}-\pi^{2} \partial_{\mu} \pi^{a}-\delta_{\mu 0} \frac{\dot{f}(t)}{f(t)} \pi^{2} \pi^{a}\right)+\mathscr{O}\left(\pi^{5}\right)
$$

with $A_{\mu}^{\mathrm{LO}}$ in (6). Thus, according to our chiral power counting, we have three types of NLO corrections to $A_{\mu \nu}$. The first is the NLO correction to the pion propagator we have evaluated in Section 3, coming from the product of the $\mathscr{O}(\pi)$ terms above. The second is the product of the $\mathscr{O}(\pi)$ with the $\mathscr{O}\left(\pi^{3}\right)$, represented by diagram c) in Fig. 2, and the third comes from the modification in $A_{\mu}$ due to the action (8), which amounts to prefactors $\left[1+f_{1}(t)\right]$ and $\left[1+f_{2}(t)\right]$ in $A_{0}$ and $A_{j}$ respectively. Then, evaluating $\rho_{\mu \nu}$ to NLO, after using (15) (we take, without loss of generality, both $t, t^{\prime} \in C_{1}$ and positive) and (22)-(23), we finally arrive to

$$
\begin{aligned}
& {\left[f_{\pi}^{s}(t)\right]^{2}=f^{2}(t)\left[1+2 f_{2}(t)-f_{1}(t)\right]-2 i G_{0}(t)} \\
& {\left[f_{\pi}^{t}(t)\right]^{2}=f^{2}(t)\left[1+f_{2}(t)\right]-2 i G_{0}(t)}
\end{aligned}
$$

for $t>0$. This is the main result of this work. It provides the NLO relationship between the PDF and $f(t){ }^{4}$. Notice that $f_{\pi}^{s}(t) \neq f_{\pi}^{t}(t)$ to NLO, unlike the equilibrium case, due to the effect of nonequilibrium renormalisation. However, note that $\left[f_{\pi}^{s}(t)\right]^{2}-\left[f_{\pi}^{t}(t)\right]^{2}=f^{2}(t)\left[f_{2}(t)-f_{1}(t)\right]$, which is finite, since it depends only on $L_{12}$, which does not renormalise. This is indeed an interesting consistency check, because the one-loop infinities appearing in $G_{0}(t)$ can then be absorbed in $L_{11}$, rendering both $f_{\pi}^{s}(t)$ and $f_{\pi}^{t}(t)$ finite. We also remark that we did not need to take $\omega_{q} \rightarrow 0^{+}$in (22)-(23) to arrive to (27)-(28) (there are still NGB to NLO). Note also that both $f_{\pi}^{s, t}$ are real to this order. We have performed the following consistency checks on (27)-(28): first, the equilibrium result (19) is recovered (for the contour $C$ ) simply by replacing $G_{0}^{e q}=-i T^{2} / 12$ and $f_{1}=f_{2}=0$. Second, by calculating $g_{\pi}(t)$ from $\rho_{S}$, through (24), we check explicitly that the WI (25) holds and, third, we have calculated $A_{\mu \nu}$ in the $\tilde{\pi}$ parametrisation, arriving to the same result.

Therefore, (27)-(28) allow to express nonequilibrium observables (like decay rates, masses, etc) to one loop in ChPT, in terms of the physical $f_{\pi}(t)$, which could be measured, for instance, in nonequilibrium lepton decays $\pi \rightarrow l \nu_{l}$. At this stage one can follow different approaches. Exact knowledge of $f(t)$ would require to solve self-consistently the plasma hydrodynamic equations or, equivalently, Einstein equations for the metric. Alternatively, one can treat $f(t)$ as external — so that (27)-(28) provide the system response — and study simple choices consistently with (4) [20]. In what follows, we shall take $f(t)$ arbitrary and expand it near $t=0^{+}$, analysing thus the short-time evolution.

For short times, the particular form of $f(t)$ is not important and we can parametrise the nonequilibrium dynamics in terms of the values of $f(t)$ and its derivatives at $t=0^{+}$. As we discussed in Section 2, this approach is justified for times $t<t_{\max }$ with $t_{\max } \simeq 1 / f_{\pi}(0) \simeq 2 \mathrm{fm} / c$ (compare to the typical plasma time scales 5-10 fm/c [12]). The general solution of (11) with KMS conditions at $t_{i}$ can be constructed in terms of two independent solutions to the homogeneous equation, which have to be continuous and differentiable $\forall t \in C$ so that the solution is uniquely defined [17]. Therefore, they have to match the equilibrium solution and its first time derivative at $t=0$. With these conditions and expanding both $f(t)$ and the solutions near $t=0^{+}$we find to the lowest order

$$
G_{0}^{11}\left(\omega_{q}, t, t\right)=-\frac{i}{2 \omega_{q}} \operatorname{coth}\left[\frac{\beta_{i} \omega_{q}}{2}\right]\left[1-m^{2} t^{2}+\mathscr{O}\left(m^{4} t^{4}\right)\right]
$$

for $t>0$, with $m^{2}=-\ddot{f}\left(0^{+}\right) / f\left(0^{+}\right)$. For $m^{2}<0$ we see the unstable modes threshold, making the pion correlation function grow with time. The effect of those modes is not important for short times though, where

\footnotetext{
${ }^{4} G_{0}(t), f_{1}(t)$ and $f_{2}(t)$ depend implicitly on $f(t)$, through (10) and (9).
} 
the exponential growth of the correlator is not appreciable. Observe that in (29) the time dependence factorises, so that the momentum dependence is the same as in equilibrium and then we can integrate it in DR, yielding the finite answer (18). Then, from (27)-(28) we get

$$
\left[f_{\pi}^{s, t}(t)\right]^{2}=\left[f_{R}^{s, t}\right]^{2}\left\{1-\frac{T_{i}^{2}}{T_{c}^{2}}+2 H t-\left[m^{2}\left(1-\frac{T_{i}^{2}}{T_{c}^{2}}\right)-H^{2}\right] t^{2}+\mathscr{O}\left(p^{3} / \Lambda_{\chi}^{2}\right)\right\}
$$

for $t>0$, where $H=\dot{f}\left(0^{+}\right) / f\left(0^{+}\right)$, with the renormalised constants

$$
\begin{aligned}
& {\left[f_{R}^{s}\right]^{2}=f^{2}\left(0^{+}\right)+4\left[\left(L_{12}-6 L_{11}\right) m^{2}-L_{12} H^{2}\right]} \\
& {\left[f_{R}^{t}\right]^{2}=f^{2}\left(0^{+}\right)+4\left[-\left(L_{12}+6 L_{11}\right) m^{2}+L_{12} H^{2}\right]}
\end{aligned}
$$

and where the $H$ and $m$ parameters (which are $\mathscr{O}(p)$ and play the role of the Hubble constant and the deceleration parameter in the Universe expansion) also get renormalised, in terms of $\dot{f}_{1,2}\left(0^{+}\right), \ddot{f}_{1,2}\left(0^{+}\right)$and so on, but those are subleading contributions. Thus, for short times, all the effect of the $S_{4}$ terms, which is $T_{i}$ independent, is to redefine $f_{\pi}\left(0^{+}\right)$, since there are no infinities coming from $G_{0}$ in DR. We insist that this is just the effect of truncating the series in $t$ and it is not true in general. Notice that (31) implies necessarily a nonzero jump $\Delta f=f\left(0^{+}\right)-f$ (see our comments in Section 2) so that the divergent part of $L_{11}$ can be absorbed in $f^{2}\left(0^{+}\right)$rendering a finite $\Delta f_{R}^{s, t}=\Delta f_{\pi}^{s, t}$. In fact, that effect is very small compared to the other contributions in (30), and so it is the difference $f_{\pi}^{s}(t)-f_{\pi}^{t}(t)$, since $L_{11}^{r}, L_{12}^{r} \simeq 10^{-3}$ [22]. Notice also that for the particular case $H^{2}=m^{2}>0$, renormalising as $f^{2}\left(0^{+}\right)=f^{2}+24 m^{2} L_{11}$ we get $f_{\pi}^{s}=f_{\pi}^{t}$ and $\Delta_{f_{\pi}}=0$.

Finally, we will estimate some physical effects related to $f_{\pi}(t)$. For that purpose, we will ignore, for simplicity, the effect of $L_{11}$ and $L_{12}$ and, based upon (19), define the plasma effective temperature as $f_{\pi}^{2}(t)=f^{2}\left[1-T^{2}(t) / T_{c}^{2}\right]$. Therefore, we can also define a critical time as $T\left(t_{c}\right)=T_{c}$ and a freezing time $T\left(t_{f}\right)=0$. Thus, we will impose $0<T(t)<T_{c}$ and then, through our short-time results for $f_{\pi}(t)$, determine either $t_{c}$ or $t_{f}$, depending on the initial conditions $(T(t)$ is just quadratic in time to this order). Notice that we are following a similar approach as in equilibrium when one extrapolates (19) until $T=T_{c}$. Let us then take typical values $T_{i},|H|,|m| \simeq 100 \mathrm{MeV}$ and retain only the leading order in $x \equiv T_{i}^{2} / T_{c}^{2}$, consistently with the chiral expansion. Then, if $H=0$, the system cools down until $t_{f}^{2} m^{2} \simeq-x(1+x)\left(t_{f} \simeq 0.2 \mathrm{fm} / c\right)$ if $m^{2}<0$, whereas for $m^{2}>0$ it is heated until $t_{c}^{2} m^{2}=1\left(t_{c} \simeq 2 \mathrm{fm} / c\right)$, independent of $T_{i}$. For $H>0$, there is cooling until $t_{f}|H| \simeq x / 2\left(t_{f} \simeq 0.2 \mathrm{fm} / c\right)$. Finally, for $H<0$ and $m^{2}>0$ there is heating until $t_{c}|H| \simeq(1-3 x / 4) / 2\left(t_{c}\right.$ $\simeq 2 \mathrm{fm} / c)$, whereas if $m^{2}<0$, there is heating until a maximum $t_{m}|H| \simeq(1+x / 2) / 2$ and then cooling down until $t_{f}|H| \simeq 1+x\left(t_{f} \simeq 2.3 \mathrm{fm} / c\right)$. We observe that the effect of the unstable modes $\left(m^{2}<0\right)$ is always to cool down the system and that the freezing time for $H<0$ is much longer than that for $H>0$. Some of these time scales are indeed longer than those to which our short-time approximation remains valid, but they have to be understood as estimates, similarly to estimating $T_{c}$ at equilibrium through (19), even though the low $T$ approach is less reliable near $T \simeq T_{c}$.

Comparing with [12], naively identifying the LSM order parameter $v(t) \simeq f_{\pi}(t)$ (in proper time), we see a similar short-time evolution, although our estimates for the time evolution duration are somewhat lower. This was expected, since the initial values in Ref. [12] correspond to $T_{i} \simeq 200 \mathrm{MeV}$ and $|H| \simeq 400 \mathrm{MeV}$, which are too high for our low-energy approach. An important remark is that in typical simulations like [12], $v(t)$ reaches a stationary value, about which it oscillates (thermalisation). It is clear that we cannot predict that type of behaviour only within our short-time approach, quadratic in time, but only estimate the time scales involved - similarly as to why ChPT cannot see the phase transition-. Therefore, in view of the above estimates, we believe that our ChPT model may be useful for studying the different nonequilibrium observables evolution, from a stage where some cooling has already taken place onwards. In principle, we could approach closer to $T_{c}$ by considering enough orders in our ChPT, although in practice, beyond one-loop, some resummation method, like large $N$, will need to be implemented. 


\section{Conclusions and Outlook}

We have extended chiral lagrangians and ChPT out of thermal equilibrium. The chiral power counting requires all time derivatives to be $\mathscr{O}(p)$ and to lowest order our model is a NLSM with $f \rightarrow f(t)$. This model accommodates unstable pion modes and corresponds to a spatially flat RW metric in conformal time with scale factor $a(t)=f(t) / f(0)$ and minimal coupling. We have exploited this analogy to establish the renormalisation procedure, which allows to construct the fourth order lagrangian absorbing all the loop divergences, which in general will be time-dependent.

We have applied our model to study the time-dependent pion decay functions, extending the equilibrium pion decay constants. In general there are two independent PDF, as in equilibrium, and to NLO in ChPT they already differ, unlike equilibrium, due to renormalisation. We have obtained them to NLO in terms of the equal-time correlation function, analysing their lowest order short-time coefficients and their dependence with $T_{i}$, and discussing the relevant time scales involved within the context of a RHIC plasma.

Among the aspects of our model which are worth to be studied further are the long-time evolution, by choosing suitable parametrisations for $f(t)$, including the analytic approach, and the behaviour of the two-point correlation function at different space points, which would allow us to investigate the formation of regions of unstable vacua (DCC) [20]. Other applications and extensions, to be explored in the future include photon production in the pion sector (by gauging the theory and including $\pi^{0}$ anomalous decay), the quark condensate time dependence (by including the mass explicit symmetry-breaking terms), the $N_{f}=3$ case, large $N$ resummation and proper time evolution.

\section{Acknowledgements}

We are grateful to T. Evans and R. Rivers for countless and fruitful discussions, as well as to R.F. Alvarez-Estrada, A. Dobado and A.L. Maroto for providing useful references and comments. A.G.N wishes to thank the Imperial College group for their kind hospitality during his stay there and has received financial support through a postdoctoral fellowship of the Spanish Ministry of Education and CICYT, Spain, project AEN97-1693.

\section{References}

[1] S. Weinberg, Physica A 96 (1979) 327; J. Gasser, H. Leutwyler, Ann. Phys. (NY) 158 (1984) 142; Nucl. Phys. B 250 (1985) 465.

[2] A. Dobado, A. Gómez Nicola, A. López-Maroto, J.R. Peláez, Effective lagrangians for the Standard Model, Springer, 1997, and references therein.

[3] F. Wilczek, Int. J. Mod. Phys. 7 (1992) 3911.

[4] A. Bochkarev, J. Kapusta, Phys. Rev. D 54 (1996) 4066.

[5] J. Gasser, H. Leutwyler, Phys. Lett. B 184 (1987) 83.

[6] P. Gerber, H. Leutwyler, Nucl. Phys. B 321 (1989) 387.

[7] A. Anselm, Phys. Lett. B 217 (1989) 169; A. Anselm, M. Ryskin, Phys. Lett. B 226 (1991) 482.

[8] K. Rajagopal, F. Wilczek, Nucl. Phys. B 399 (1993) 395.

[9] D. Boyanowsky, H.J. de Vega, R. Holman, Phys. Rev. D 51 (1995) 734.

[10] D. Boyanowsky et al., Phys. Rev. D 56 (1997) 3929.

[11] A.J. Gill, R.J. Rivers, Phys. Rev. D 51 (1995) 6949.

[12] F. Cooper, Y. Kluger, E. Mottola, J.P. Paz, Phys. Rev. D 51 (1995) 2377; M.A. Lampert, J.F. Dawson, F. Cooper, Phys. Rev. D 54 (1996) 2213.

[13] A. Barducci et al., Phys. Lett. B 369 (1996) 23.

[14] R. Baier et al., Phys. Rev. D 56 (1997) 2548, 4344; M. Le Bellac, H. Mabilat, Z. Phys. C 75 (1997) 137.

[15] J. Schwinger, J. Math. Phys. 2 (1961) 407; L.V. Keldysh, Sov. Phys. JETP 20 (1965) 1018.

[16] K.C. Chou, Z.B. Sou, B.L. Hao, L. Yu, Phys. Rep. 118 (1985) 1. 
[17] G. Semenoff, N. Weiss, Phys. Rev. D 31 (1985) 689.

[18] D. Boyanowsky, D.S. Lee, A. Singh, Phys. Rev. D 48 (1993) 800.

[19] M. Le Bellac, Thermal Field Theory, Cambridge U.P., 1996.

[20] A. Gómez Nicola, work in progress.

[21] N. Birell, P. Davies, Quantum Fields in Curved Space, Cambridge U.P., 1982.

[22] J.F. Donoghue, H. Leutwyler, Z. Phys. C 52 (1991) 343.

[23] R.D. Pisarski, M. Tytgat, Phys. Rev. D 54 (1996) R2989. 